\title{
The Journal of Youth and Adolescence at 40
}

\author{
Roger J. R. Levesque
}

Published online: 27 November 2010

(C) Springer Science+Business Media, LLC 2010

This issue marks yet another transition in the life course of the Journal of Youth and Adolescence: its 40th volume. The Journal's 40-year history also happens to mark the birth and growth of the modern empirical study of adolescence (for a brief review of the journal's history, see Levesque 2007a). The journal, under the leadership of its founding editor, Daniel Offer, played an important role in shepherding the field from its infancy to what it is today (see Levesque 2007b, 2008). As with the study of adolescence itself, the journal has grown tremendously over the past few years. Indeed, it seems to have hit a growth spurt over the past five, when the journal went from 6 issues per year to 12 and experienced a parallel leap in the number of articles per issue.

Given the above developments, it is not surprising to find that the journal remains the most cited to journal broadly devoted to the study of adolescence. According to 2009 Journal Citation Reports, for example, these were the total citations to journals publishing broadly in this area: Journal of Youth of Adolescence, 2,602 citations; Journal of Adolescence, 2,271 citations, Adolescence, 1,611 citations; Journal of Adolescent Research, 1,361 citations; Journal of Research on Adolescence, 1,253 citations; Journal of Early Adolescence, 1,062 citations; and Youth and Society, 678 citations. Alternatively, and given that some journals do not have as long a history, the breadth of citations can be assessed in terms of cited to articles from recent years (here 2007 and 2008) in a given year (here 2009); and doing so does not change much the relative citations to journals: Journal of Youth of Adolescence, 249 citations; Journal of Adolescence, 218 citations,

R. J. R. Levesque ( $\square)$

Indiana University, 302 Sycamore Hall, Bloomington, IN, USA

e-mail: rlevesqu@indiana.edu
Adolescence, 83 citations; Journal of Adolescent Research, 80 citations; Journal of Research on Adolescence, 111 citations; Journal of Early Adolescence, 57 citations; and Youth and Society, 48 citations. The journal remains the most highly cited to journal of its kind. Of course, we may quibble about ranking efforts, as recently done in other fields where editors have argued for taking more seriously recent, overall citations to journals in addition to Impact Factors that are ratios of the number of cited to articles to the number of those published within a given period (e.g., Marabia and Constanza 2008). We also may want to include other journals that are not focused solely on adolescence (Developmental Psychology or Child Development) or those that include particular aspects of adolescent development (Journal of Adolescent Health or Development and Psychopathology). Regardless of these approaches, it is clear that the journal has had an impressive footprint on the study of adolescence. It also is clear that the journal shares its influence with other journals and that it plays a key role in the impact of those journals by so heavily citing to them.

The intense growth in research on adolescence contributed to important developments for the journal. Most notably, the high volume of submissions has resulted in an increasing percentage of rejected manuscripts. More importantly for the journal, however, the higher rejection rate also comes from a move away from publishing studies that focus on the development of measures, literature reviews that lack an empirical component, and qualitative studies. These developments have allowed the journal to grow while still relying mainly on the editorial board rather than on other external reviewers, a reliance that has helped maintain consistency in reviews and accepted manuscripts. The changes also have allowed us to return to publishing book reviews (see Levesque 2007c), special issues (see, 
e.g., Lerner et al. 2010; Levesque 2010, Horn et al. 2009), invited commentaries (Walsh and Donaldson, 2009; Ballard et al. 2010; Markey 2010) as well as letters to the editor (see Pokhrel et al. 2010). These changes have allowed us to give readers a broader sense of cutting-edge developments in the study of adolescence.

Despite some of the above changes, it is important to emphasize that the journal remains firmly committed to showcasing empirical studies. The journal's mission still centers on empirical analyses that take developmental sciences seriously and that closely consider the implications of their findings for youth (see Levesque 2007a). As a result, we continue to publish studies from a very wide variety of disciplines that highlight key issues facing youth. Over the past 5 years, for example, we have published well over 500 empirical articles. A look at those articles reveals that they have focused on the typically identified important aspects of adolescent life, such as families, peers, sexuality, deviancy, communities, media, mental health, aggression, schooling, physical development, thriving, and a wide variety of harms (e.g., suicide). What may be less obvious, but equally important, is the extent to which these studies have emphasized diversity in terms of, for example, gender, sexual orientation, social class, ethnicity, race, religion, ability, age, and nationality. In addition to emphasizing diversity, articles have focused on how to approach diversity (such as through understanding the nature and effects of discrimination), how social systems respond to youth in need (such as through juvenile justice systems, child welfare systems and medical systems), as well as how social systems (such as schools, families and peers) can exacerbate or help address youth's needs. In these efforts, I remain grateful to Judy Jones, Springer's Senior Editor for Behavioral Sciences, who has helped the journal smoothly transition its editorship and embrace the electronic age. I also, of course, remain grateful for the trust that authors continue to place in our journal. Our editorial team continues to look forward to serving authors and readers committed to understanding adolescents.

\section{References}

Ballard, E., Bosk, A., \& Pao, M. (2010). Invited commentary: Understanding brain mechanisms of pain processing in adolescents' non-suicidal self-injury. Journal of Youth and Adolescence, 39, 327-334.
Horn, S. S., Kosciw, J. G., \& Russell, S. T. (2009). Special issue introduction: New research on lesbian, gay, bisexual, and transgender youth: Studying lives in context. Journal of Youth and Adolescence, 38, 863-866.

Lerner, R. M., von Eye, A., Lerner, J. V., Lewin-Bizan, S., \& Bowers, E. P. (2010). Special issue introduction: The meaning and measurement of thriving: A view of the issues. Journal of Youth and Adolescence, 39, 707-719.

Levesque, R. J. R. (2007a). Reporting empirical research on adolescence: Reflections on the appeal of coherence, purpose and responsibility. Journal of Youth and Adolescence, 36, 1-9.

Levesque, R. J. R. (2007b). Reflections on a legendary contribution to the empirical study of adolescence. Journal of Youth and Adolescence, 36, 1-4.

Levesque, R. J. R. (2007c). Book reviews and the need to take books more seriously. Journal of Youth and Adolescence, 36, 10861088.

Levesque, R. J. R. (2008). Introduction to special section: Continuing our tribute to dan offer. Journal of Youth and Adolescence, 36, $1161-1162$.

Levesque, R. J. R. (2010). Special issue introduction: The place of self-harm in adolescent development. Journal of Youth and Adolescence, 39, 217-218.

Marabia, A., \& Constanza, M. C. (2008). Don't step on our 2007 citation footprint (CF). Preventive Medicine, 47, 351-353.

Markey, C. N. (2010). Invited commentary: Why body image is important to adolescent development. Journal of Youth and Adolescence, 39, 1387-1391.

Pokhrel, P., Brown, B. B., Moran, M. B., \& Sussman, S. (2010). Letter to the editor: Comments on adolescent peer crowd affiliation: A response to cross and fletcher (2009). Journal of Youth and Adolescence, 39, 213-216.

Walsh, S. M., \& Donaldson, R. E. (2009). Invited commentary: National safe place: Meeting the immediate needs of runaway and homeless youth. Journal of Youth and Adolescence, 39, $437-445$.

\section{Author Biography}

Roger J. R. Levesque is Chair and Professor of Criminal Justice, Indiana University. He is Editor of the Journal of Youth and Adolescence. 